\title{
Investigação sobre os Fatores de Risco da Prematuridade: uma Revisão Sistemática
} Research on the Risk Factors of Prematurity: A Systematic Review

\author{
THASSIANY SARMENTO OLIVEIRA DE ALMEIDA ${ }^{1}$ \\ RAQUEL PINTO LINS ${ }^{2}$ \\ ADEMIR DE LIMA CAMELO \\ DANDARAH CHRISTIE CAVALCANTI LIMA DE MELLO ${ }^{3}$
}

\section{RESUMO}

Objetivo: avaliar os fatores de risco da prematuridade ou o baixo peso em decorrência do parto prematuro. Para tanto realizou-se uma revisão sistemática. Material e Métodos: Foram selecionados, por meio de busca eletrônica, artigos das bases de dados MEDLINE, LILACS e SciELO publicados de 2005 a 2011. Para o levantamento foram utilizados os descritores prematuridade e premature birth. A força de associação entre o objetivo de cada artigo e o objetivo desta revisão foi avaliada baseada nos seguintes critérios: relação temporal, validade interna, força de associação e plausibilidade biológica da associação. Resultados: após a leitura dos resumos dos 122 artigos selecionados, foram selecionados 38 para a análise, incluindo-se apenas 10 na presente revisão. $O$ baixo peso ao nascer foi analisado em associação com a prematuridade $(n=9)$ artigos. A busca por artigos para realizar esta revisão demonstrou a escassez de estudos variados que objetivaram analisar a relação entre prematuridade e os riscos para o desenvolvimento de doenças em crianças ou a cronicidade em adultos. Conclusão: a prematuridade consiste em um tema de interesse bastante recente.

\section{DESCRITORES}

Doenças do Prematuro. Trabalho de Parto Prematuro. Prematuro.

\begin{abstract}
Objective: To evaluate, by means of a systematic review, the risk factors of prematurity or low birth weight due to premature birth. Material and Methods: We carried out searches of articles in the databases MEDLINE, LILACS and SciELO published from 2005 to 2011, using the descriptors prematurity and premature birth. The strength of association between the objective of each article and the purpose of this review was evaluated based on the following criteria: temporal relationship, internal validity, strength of association, and biological plausibility of the association. Results: After reading the abstracts of the 122 articles retrieved, 38 were selected for analysis, of which 10 were included in this review. Low birth weight was analyzed in association with prematurity $(n=9)$. The findings of this review demonstrated a lack of varied studies that aimed to analyze the relationship between prematurity and risk for developing diseases in children or chronicity in adults. Conclusion: prematurity consists of a recent topic of interest.
\end{abstract}

\section{DESCRIPTORS}

Infant, Premature, Diseases. Obstetric Labor, Premature. Infant, Premature.

\footnotetext{
Mestranda em Saúde Pública pela Universidade Estadual da Paraíba (UEPB),Campina Grande/PB, Brasil.

2 Estudante de graduação em Medicina pela Faculdade de Ciência Médicas (FCM), Campina Grande/PB, Brasil.

3 Graduanda em Odontologia pela Universidade Estadual da Paraíba (UEPB), Campina Grande/PB, Brasil.
} 
$\mathrm{O}$ termo prematuridade é definido, segundo a Organização Mundial de Saúde (OMS, 1961), como o nascimento abaixo de 37 semanas de gestação. Ele pode ser classificado em prematuridade moderada (32 semanas a 36 semanas de idade gestacional), prematuridade acentuada (28 semanas a 31 semanas de idade gestacional) e prematuridade extrema (inferior a 28 semanas de idade gestacional) (LORENA,BRITO, 2009).

A sobrevida de Recém-Nascidos Pré-Termo (RNPT) vem tornando frequente a ocorrência de morbidade, retinopatia da prematuridade, displasia broncopulmonar, apneia da prematuridade, entre outras enfermidades (SINCLAIR, BRACKEN, ATKINSON, 1992). Alguns estudos, realizados nas últimas décadas, evidenciaram associação entre prematuridade, condição de crianças nascidas com Idade Gestacional (IG) inferior a 37 semanas, e o desenvolvimento de alguns agravos como hipertensão arterial, intolerância à glicose e dislipidemias tanto em crianças (CHEUNG et al., 2004) como em adultos (DALZIEL et al., 2007). A importância do estudo da prematuridade deve-se à elevação dos números de incidência de morbiletalidade neonatal, além dos altos custos das internações e ocorrência de sequelas de múltiplas naturezas (LORENA,BRITO, 2009).

Em 2011 foi publicado um artigo que objetivou apresentar o com-portamento de algumas variáveis epidemio-lógicas e clínicas, relacionadas à prematuridade. Trata-se de um estudo descritivo e retrospectivo, no qual foram avaliados 189 prontuários clínicos de mulhe-res que tiveram partos no Hospital Geral de Itapeceri-ca da Serra em São Paulo (Brasil), e que aten-deram aos seguintes critérios de inclusão: partos de qualquer tipo ocorridos no ano selecionado; IG entre 22 e 36 semanas e seis dias, avaliada pelo método de Capurro ou New Ballard(RABELLO, BARROS, 2011). Após o nascimento $54,5 \%$ das crianças foram encaminhadas para a UTI neonatal. Dentre os óbitos neonatais ocorridos, três ocorreram com idade gestacional en-tre 25 e 27 semanas de gestação, caracterizando a pre-maturidade extrema e mortalidade. A prematuridade foi referida no trabalho, ainda, como sendo responsável por graves danos imediatos nos Recém-Nascidos (RN) e por sequelas tardias (RABELLO, BARROS, 2011).

A cada dia nascem, no mundo, cerca de 13 milhões de prematuros (10\% dos nascimentos), de acordo com a revisão sobre o tema publicada no Bulletin of the World Health Organization em 2010 (BECK et al., 2010). Em hospitais de atendimento obstétrico, como o Hospital Geral de Itapeceri-ca da Serra, a prevalência de prematuridade no local do estudo foi de $7,8 \%$ do total de partos no período de um ano do estudo (RABELLO, BARROS, 2011). Segundo o Ministério de saúde (BRASIL, 2010), a proporção de nascidos vivos prematuros permanece estável no Brasil desde 2000, apresentando uma taxa média de $6,6 \%$. O desenvolvimento de unidades de terapia intensiva para cuidados neonatais aumentou a taxa de sobrevida de prematuros. Entretanto, mesmo com o avanço da tecnologia voltada para essa área médica, os efeitos da prematuridade ao longo da vida são pouco conhecidos. Mais escasso ainda é o entendimento da relação de caso/consequência do nascimento prematuro sobre as doenças crônicas em fases posteriores da vida (DALZIEL et al., 2007).

O emprego de uma abordagem multidisciplinar o mais precocemente possível se faz necessária para amenizar ou prevenir sequelas. As medidas preventivas adotadas no período neonatal envolvem todos os cuidados neonatais oferecidos ao RNPT e não são facilmente estabelecidas, uma vez que a fisiopatologia é complexa e multifatorial, sendo os RN amiúde assintomáticos. A questão é que as pessoas nascidas prematuras estão mais sujeitas aos agravos futuros advindos da própria condição da prematuridade, como consequência da pouca maturidade de órgãos e danos advindos do baixo peso ao nascer associado (COELLI et al., 2011). Levando em consideração os resultados de pesquisas realizadas com crianças nascidas prétermo e de evidências da possível associação de doenças com a prematuridade, o presente estudo teve como objetivo realizar uma revisão sistemática sobre esta temática.

\section{MATERIAL E MÉTODOS}

Foram selecionados, por meio de busca eletrônica, artigos das bases de dados MEDLINE, LILACS e SciELO publicados de 2005 a 2011. Para o levantamento foram utilizados os seguintes descritores: prematuridade e premature birth. Os termos descritores foram utilizados separadamente no campo de busca de cada base de dados, sendo utilizadas as ferramentas de refinamento quando estas estavam disponíveis, como nas bases MEDLINE e LILACS. Nas bases de dados analisadas, os artigos foram limitados pelo ano de publicação e selecionados posteriormente de acordo com os temas e a população estudada.

Para serem anexadas à análise, as pesquisas deveriam ter sido planejadas para avaliar os fatores de risco da prematuridade ou o baixo peso em decorrência do parto prematuro, assim como deveriam preencher outras condições tais como: estudo epidemiológico 
(descritivo, série de casos, transversal, caso-controle ou coorte) ou experimental que enfocasse os riscos do nascimento pré-termo. Os critérios de exclusão para a seleção dos artigos foram: (1) artigos que não apresentavam resumo, (2) artigos de revisão, (3) artigos indisponíveis em português, inglês ou espanhol e (4) artigos que abordavam essencialmente mortalidade neonatal, manifestações perinatais ou relação mãe/bebê prematuro.

Após a leitura dos resumos dos 122 artigos selecionados, foram excluídos 84 por não se adequarem a presente pesquisa. Assim, foram excluídos os artigos que não apresentassem em sua análise nenhuma proposta de verificar os fatores de risco decorrentes da prematuridade. Ao final da busca foram selecionados para esta análise 38 estudos. Após a leitura dos 38 artigos foram excluídos 28 da análise, pois não relacionaram a influência da variável prematuridade sobre a doença abordada. Dos 10 artigos restantes, um referia-se ao estudo clínico-epidemiológico, três tratavam-se de estudo de coorte, dois eram estudos transversais, um caso-controle e três utilizaram abordagem descritiva, conforme apresentado na Tabela 1. Para cada artigo foram extraídos detalhes quanto à população de estudo, ao tipo de estudo, ao local e à exposição ao fator de risco (prematuridade). A força de associação entre o objetivo de cada artigo e o objetivo desta revisão foi avaliada baseada nos seguintes critérios: relação temporal, validade interna, força de associação e plausibilidade biológica da associação.

\section{DISCUSSÃO TEÓRICA}

Na Tabela 1 encontram-se as características gerais dos dez estudos utilizados na presente análise, incluindo tipo de estudo, ano de publicação, amostra, critério para a classificação de prematuridade e ferramenta utilizada para desenvolvimento da pesquisa. Apenas três artigos utilizaram ferramentas - CARLINO et al.,(2010), FELICE et al., (2010)e TIAGO, CALDEIRA, VIEIRA, (2008). Com exceção da pesquisa de JOHANSSON et al., (2005), e LOMUTO et al.,(2010), todos os estudos foram realizados no Brasil, sendo que cinco foram conduzidos apenas com RN e os outros cinco com crianças. $\mathrm{O}$ baixo peso ao nascer foi analisado em associação com a prematuridade em todos os artigos, exceto no trabalho de JOHANSSON et al.,(2005), onde objetivou-se analisar a associação entre prematuridade e risco para desenvolver pressão alta em jovens adultos.

$\mathrm{Na}$ literatura encontram-se poucos estudos que têm abordado a prematuridade como fator de risco à saúde na vida adulta, principalmente pela necessidade de se acompanhar, por um grande período de tempo, o grupo de indivíduos. Embora seja de difícil condução, alguns desses estudos têm apontado a associação entre o tempo de gestação e o aumento do risco para o desenvolvimento de doenças cardiovasculares e outros agravos crônicos. JOHANSSON et al.,(2005), desenvolveram um estudo na Suécia, com uma grande amostra de homens, identificando que o nascimento prematuro constitui fator de risco para hipertensão arterial e este risco torna-se particularmente elevado nos homens que nasceram com, pelo menos, oito semanas antes do tempo esperado. Homens nascidos antes de 29 semanas de gestação tiveram risco duas vezes maior de apresentar Pressão Arterial Sistólica elevada (PAS e" 140/90 mmHg). Por sua vez, os nascidos entre 29 e 32 semanas e aqueles entre a $33^{\mathrm{a}}$ e a $36^{\mathrm{a}}$ semanas de gestação apresentaram, respectivamente, $48 \%$ e $24 \%$ mais chances de apresentarem PAS elevada. A associação entre IG e PAS prevaleceu mesmo depois de controlado o fator familiar como idade da mãe ao nascimento, níveis educacional e socioeconômico familiar (JOHANSSON et al., 2005).

A relação entre o baixo peso ao nascer e o desenvolvimento de hipertensão arterial essencial em fases posteriores da vida tem sido mais estudada do que a relação entre IG e hipertensão arterial. JOHANSSON et al., (2005), afirmam, porém, que a alta mortalidade em prematuros e as deficiências metodológicas, como a falta de ajuste para o peso atual, podem ter levado à superestimação da relação entre peso ao nascer e alterações da pressão arterial ao longo vida.

O nascimento de crianças de baixo peso constitui um dos grandes desafios aos serviços do SUS, não somente por elas demandarem maiores custos hospitalares, mas também, por apresentarem maior morbidade ao longo da vida, com risco aumentado de distúrbios metabólicos, déficits neurológicos e diminuição da capacidade cognitiva (TIAGO, CALDEIRA, VIEIRA, 2008). As políticas e práticas em saúde pública para redução do risco de baixo peso ao nascimento são implantadas a partir do conhecimento dos fatores de risco associados, tais como tabagismo materno e prematuridade(TIAGO, CALDEIRA, VIEIRA, 2008). Diante do exposto se justificam os estudos epidemiológicos que buscam identificar os fatores de risco para a ocorrência do baixo peso ao nascer, e a importância relativa à prematuridade. Em um hospital público de referência da região norte de Minas Gerais, promoveram um estudo descritivo e retrospectivo, com 510 crianças nascidas entre julho de 2003 e junho de 2005 e selecionadas mediante aplicação de cálculo amostral. Do total de crianças $16,4 \%$ eram prematuras. 
Tabela 1: Caracteristica dos artioos selecionados para a revisão

\begin{tabular}{|c|c|c|c|c|c|}
\hline Autor & Tipo de estudo & Local (ano) & Amostra & Prematuridade & Ferramenta \\
\hline Argollo at al. & $\begin{array}{l}\text { Estudo dínico- } \\
\text { epidemiológico } \\
\text { com base em } \\
\text { dados } \\
\text { secundários. }\end{array}$ & Brasil (2006) & $\begin{array}{l}\mathrm{N}=93 \mathrm{RN} \text { com } \\
\text { sinais de LSB ao } \\
\text { ultra-som. }\end{array}$ & $\begin{array}{c}(1)<32 \\
\text { samanas; }(2) \\
32,0 \text { a } 33,6 ;(3) \\
34,0 \text { até } 36,6 .\end{array}$ & \\
\hline Carlino ef al & Caso-controle. & Brasil (2010) & $\begin{array}{c}N=40 \text { crianças } \\
\text { de idada } \\
\text { cronolbgica de } \\
12 \text { a } 24 \text { meses. }\end{array}$ & $\begin{array}{l}\text { (G1) } 20 \text { criancas, } \\
\text { prematuras e } \\
\text { de baixo peso; } \\
\text { (G2) } 20 \text { crianças } \\
\text { a termo e com } \\
\text { peso } 25009\end{array}$ & $\begin{array}{l}\text { ELM e entrevista } \\
\text { semiestruturada. }\end{array}$ \\
\hline Caixata e Comea. & Estudo descritivo. & Brasi (2005) & $\begin{array}{l}\mathrm{N}=100 \text { crianças } \\
\text { prematuras, } \\
\text { entre } 6 \text { meses a } \\
6 \text { anos de idade. }\end{array}$ & $\begin{array}{l}\text { Nascimertos } \\
\text { com mais de } 20 \\
\text { semanas } e \\
\text { mencs de } 37 \text {. }\end{array}$ & \\
\hline Felice of aí. & Estudo transversal. & Brasil $(2010)$ & $\begin{array}{c}N=7 \text { lactentas } \\
\text { nascidas pré- } \\
\text { termo. }\end{array}$ & $\begin{array}{l}\text { (1) } 3 \text { crianças } \\
\text { com idade } \\
\text { corrigida de } \\
7 \text { mases (2) } 4 \\
\text { com } 8 \text { meses de } \\
\text { idade corricida. }\end{array}$ & $\begin{array}{c}\text { Escala de } \\
\text { Deservolvimento } \\
\text { de Denwer }\end{array}$ \\
\hline Hentges ot al. & $\begin{array}{l}\text { Estudo de coote } \\
\text { prospectivo. }\end{array}$ & Brasil (2010) & $\begin{array}{c}\mathrm{N}=87 \mathrm{RN} \text { com } \\
\text { cafeina } \\
\text { detectávet, } \mathrm{N}= \\
40 \mathrm{RN} \text { sam } \\
\text { cafeina } \\
\text { detectável. }\end{array}$ & $\begin{array}{l}1 G=34 \\
\text { semanas }\end{array}$ & \\
\hline Johansson af al. & Estudo de coorte. & $\begin{array}{l}\text { Suécia } \\
(2005)\end{array}$ & $\begin{array}{c}\mathrm{N}=329495 \\
\text { homens } \\
\text { nascidos entre } \\
1973 \text { e } 1981 \text { que } \\
\text { se cadastraram } \\
\text { no serviço mitar } \\
\text { de } 1993 \text { a } 2001\end{array}$ & $\begin{array}{l}\text { (1) Nascidos } \\
\text { antes de } 29 \\
\text { semanas de IG; } \\
\text { (2) Nascidos } \\
\text { entre } 29 \text { e } 32 \text {; (3) } \\
\text { Nascidos apos } \\
32 \text { semanas. }\end{array}$ & \\
\hline Lima ef al. & $\begin{array}{l}\text { Estudo de coote } \\
\text { retrospectivo. }\end{array}$ & Brasil (2007) & $N=818 R N$ & $\begin{array}{l}1 G^{-3} \cdot 37 \\
\text { semanas }\end{array}$ & \\
\hline Lamuto ef al. & $\begin{array}{l}\text { Estudo descritivo e } \\
\text { retrospectivo. }\end{array}$ & $\begin{array}{c}\text { Argentina } \\
(2010)\end{array}$ & $\begin{array}{l}\mathrm{N}=3371 \mathrm{RN} \text { de } \\
\text { risco de } \\
\text { apresentar ROP }\end{array}$ & $\begin{array}{l}\text { (1) RNPT com } \\
\text { peso? } 1500 \mathrm{~g} \text { ou } \\
\text { IG = que } 32 \\
\text { semanas. (2) RN } \\
\text { de peso? } 1500 \mathrm{~g} \\
\text { ou IG de } 33 \text { a } 36 \\
\text { semanas que } \\
\text { receberam } \\
\text { oxigenioterapia }\end{array}$ & \\
\hline Nascimerto. & $\begin{array}{c}\text { Estudo transversal, } \\
\text { com base em } \\
\text { dados } \\
\text { secundárics. }\end{array}$ & Brasi (2008) & $\begin{array}{c}\mathrm{N}=33653 \mathrm{RN} \\
\text { vivos }\end{array}$ & $\begin{array}{l}1 \mathrm{G} ? 37 \\
\text { semanas. }\end{array}$ & \\
\hline Tiago af al. & $\begin{array}{l}\text { Estudo descritivo e } \\
\text { retrospectivo. }\end{array}$ & Brasi (2008) & $\mathrm{N}=510$ crianças & $\begin{array}{l}\text { IG? } 37 \\
\text { semanas }\end{array}$ & $\begin{array}{c}\text { Formulário de } \\
\text { anâlise } \\
\text { documental. }\end{array}$ \\
\hline
\end{tabular}


O estudo mostrou associação do baixo peso ao nascer com a prematuridade $(\mathrm{OR}=108,90)$ (TIAGO, CALDEIRA, VIEIRA, 2008).

Os defeitos do esmalte do dente e a erupção dentária em crianças nascidas prematuras foram relatados no artigo de CAIXETA E CORRÊA,,(2005). Os autores realizaram um estudo descritivo com 100 crianças diagnosticadas como prematuras, segundo a OMS, na faixa etária compreendida entre seis meses e seis anos de idade no Instituto da Criança do Hospital das Clínicas da Faculdade de Medicina da Universidade de São Paulo (FMUSP). Para análise da dentição decídua, os autores consideraram que a erupção dentária ocorre em média por volta do sexto mês de vida, completandose no terceiro ano de vida. $\mathrm{O}$ estudo propôs-se, ainda, a avaliar o início da dentição permanente com a erupção do primeiro molar por volta do sexto ano de vida, já que o início da formação deste dente ocorre entre o terceiro e quarto mês de vida intrauterina e o início de sua mineralização, ao nascimento. Para a coleta dos dados, desenvolveram uma avaliação médica e odontológica. Após a análise dos dados, os resultados mostraram uma frequência de $35 \%$ de crianças com defeitos do esmalte e $65 \%$ sem defeitos. Em relação ao baixo peso, verificouse o peso médio de $1.672 \mathrm{~g}$ para o grupo com defeito e de $1.855 \mathrm{~g}$ para as crianças sem defeito de esmalte. Opacidades brancas, aparecendo na dentição decídua com maior frequência no canino inferior $(6,74 \%) \mathrm{e}$ incisivo lateral superior $(4,49 \%)$, e as opacidades amarelas, nos incisivos centrais superiores e segundo molar superior $(2,24 \%)$, foram os defeitos mais comuns relatados no trabalho. Os dentes hipoplásicos também foram referidos. Verificaram, ainda em relação à erupção, que $42 \%$ das crianças tiveram seu primeiro dente erupcionado entre o $6^{\circ}$ e o $10^{\circ}$ mês de vida, $36 \%$ entre o $10^{\circ}$ e o $18^{\circ}$ mês e $3 \%$ até cinco meses. Foram encontrados diversos trabalhos que enfocaram a prematuridade como uma das causas do aparecimento de defeitos do esmalte e atraso na erupção dentária. A associação entre a prematuridade e o baixo peso foi descrita como uma das causas do aparecimento de defeitos do esmalte e atraso na erupção dentária, ao apontarem que à medida que o RN apresentava peso mais próximo da normalidade o defeito no esmalte evidenciava-se menor (CAIXETA E CORRÊA, 2005).

Indubitavelmente o nascimento prematuro representa um fator de risco para o desenvolvimento motor adequado de lactentes, porque, frequentemente, associa-se a problemas neonatais, tais como: hemorragia intraventricular, hipóxia, baixo peso ao nascimento e outras intercorrências neurológicas, que de acordo com a intensidade e duração podem resultar em sequelas permanentes que no futuro irão interferir na vida do bebê(FELICE et al., 2010).

Segundo a literatura, regiões do cérebro são altamente vulneráveis a danos por hipóxia e injúrias periventrivulares, como a leucomalácia periventricular frequentemente associada à prematuridade e ao baixo peso, o que pode contribuir para a elevada incidência de desordens da linguagem (CARLINO, LAMÔNICA, ALVARENGA, 2010). A defasagem verificada no desenvolvimento de RNPT pode estar associada não necessariamente com lesões cerebrais, mas pode significar imaturidade do sistema nervoso central, que pode funcionar como indicador de desordens do desenvolvimento. Crianças pré-termo e de baixo peso apresentam provável risco de desenvolver alterações de linguagem por atraso ou distúrbios nos processos receptivos, expressivos envolvendo todos os níveis linguísticos, além dos cognitivos, sensoriais e perceptivos (CARLINO, LAMÔNICA, ALVARENGA, 2010).

CARLINO, LAMÔNICAEALVARENGA, (2010), publicaram um estudo que envolveu 40 crianças de idade cronológica de 12 a 24 meses, divididas em dois grupos: G1 (20 crianças com idade gestacional variando de 22 a 34 semanas e de baixo peso ou de muito baixo peso) e G2 (20 crianças a termo com peso superior a 2500g). Através da aplicação da escala Early Language Milestone Scale (ELM) estabeleceram a comparação do desempenho dos grupos nas três áreas que se mostraram estatisticamente diferentes: área auditiva receptiva $(\mathrm{p}=0,0002)$, auditiva expressiva $(\mathrm{p}=0,0088)$ e visual $(\mathrm{p}=0,0327)$. Os escores confirmaram dados da literatura ao considerar que a prematuridade associada ao baixo peso são fatores de risco para atraso do desenvolvimento da linguagem. As crianças prematuras e de muito baixo peso apresentaram acentuados prejuízos nessas áreas avaliadas (CARLINO, LAMÔNICA, ALVARENGA, 2010).

O atraso ou alteração no desenvolvimento motor, comuns em bebês pré-termo, pode fazer com que estes percam oportunidades concretas de acrescentar aos seus repertórios, ocasionando lacunas na área perceptiva, cognitiva, linguística e social, interferindo, destarte, no desenvolvimento geral da criança (CARLINO, LAMÔNICA, ALVARENGA, 2010). Contudo, deve-se ressaltar de que o desenvolvimento da linguagem pode se encontrar defasado mesmo que a criança pré-termo tenha apresentado desenvolvimento motor adequado.

Considerando a prematuridade um fator de risco para o desenvolvimento motor, e o impacto do prejuízo das atividades apendiculares, foi desenvolvido um estudo que buscou avaliar, mediante aplicação do teste 
de desenvolvimento de Denver, a função e o desempenho apendicular de lactentes pré-termos, internados em unidade de terapia intensiva neonatal de um hospital conveniado ao SUS na cidade de Dourados - MS (Brasil). FELICE et al., (2010) recrutaram sete lactentes pré-termo com idade corrigida de sete meses (53\%) e oito meses (47\%), com IG acima de 33 semanas e peso ao nascimento entre 1.500 a 2.500 g que não apresentavam déficits neurológicos e sensoriais ou malformações. Todos os pacientes realizaram atividades para avaliar a motricidade fina e grossa. Três dos sete itens executados pelas crianças com sete meses de idade corrigida, não apresentaram resultados positivos, suspeitando-se de atraso motor (principalmente no que concerne a motricidade fina) em decorrência do pobre controle postural. Os lactentes com oito meses de idade corrigida não obtiveram sucesso na realização de apenas um item (50\% dos lactentes com oito meses de idade corrigida), não apresentando, portanto, suspeita de alteração apendicular (FELICE et al., 2010).

Diversas condições particulares da prematuridade podem prejudicar o desenvolvimento da função visual, considerado fundamental para o desenvolvimento global da criança (CARLINO, LAMÔNICA, ALVARENGA, 2010). Bebês prematuros podem sofrer com variadas alterações oftalmológicas, como estrabismo, erros de refração ou retinopatias. Em um estudo observacional, descritivo e retrospectivo desenvolvido na Argentina, a partir de informações recebidas de 24 serviços de neonatologia, LOMUTO et al., (2010), incluíram todos os RNPT com peso inferior a $1500 \mathrm{~g}$ ou IG d" que 32 semanas; também incluíram os RN de peso > que $1.500 \mathrm{~g}$ ou IG de 33 a 36 semanas que receberam oxigenioterapia no período neonatal com qualquer risco de desenvolver Retinopatia da Prematuridade (ROP) de forma precoce. Do total de pacientes de risco, 3.371 (mediana 95,9\%) foram avaliados pelo oftalmologista. 11,93\% (362) crianças apresentaram algum grau de ROP. Dos prematuros com peso inferior a $1.500 \mathrm{~g}(956), 26,25 \%$ (251) desenvolveram esta patologia. Foram tratadas 79 crianças cada uma em seu próprio hospital ou cidade. A média de peso ao nascer entre os tratados foi de $1.156 \mathrm{~g}$ e a média de $1070 \mathrm{~g}$, sendo a média e a mediana da IG de 29 semanas (RI: 2731) (LOMUTO et al., 2010).

Para LOMUTO et al., (2010), a quantidade de crianças diagnosticadas com algum grau de ROP, especialmente aqueles menores de $1.500 \mathrm{~g}$ de peso ao nascer, duplica em relação ao número de detectados no países desenvolvidos. Eles concluíram que os serviços públicos avaliados do país (Argentina), continuam vivendo a "terceira epidemia de retinopatia da prematuridade" (característica dos países em desenvolvimento, com elevado crescimento neonatal e deficiências na qualidade de atenção), com proporções importantes de crianças afetadas e tratadas.

O papel do consumo materno de cafeína e a ocorrência de apneia da prematuridade em RNPT têm suscitado interesse dos profissionais de saúde em geral, uma vez que a cafeína ao atravessar a barreira placentária pode estimular o centro respiratório fetal (GROSSO et al., 2008). Em um estudo de coorte prospectivo, conduzido no Hospital de Clínicas de Porto Alegre (Brasil) por HENTGES et al., (2010), a amostra foi composta de RNPT com peso de nascimento entre 1.000 e $2.000 \mathrm{~g}$ e IG menor que 37 semanas. Os RN foram divididos em dois grupos: O Grupo (1) envolvia 87 nascidos pré-termo com cafeína detectável no sangue de cordão umbilical, enquanto o Grupo (2) era formado por 40 RNPT com cafeína indetectável no sangue de cordão umbilical. Este trabalho mostrou que tanto os recém-nascidos pré-termo com níveis detectáveis de cafeína nos sangue do cordão umbilical quanto os com níveis indetectáveis apresentaram ocorrência similar de apneia; e entre os que apresentaram apneia, os que tinham níveis de cafeína detectável no sangue de cordão manifestaram apneia mais tardiamente. No grupo sem cafeína detectável no sangue de cordão umbilical, a IG foi significativamente menor $(p=0,009)$, e eles necessitaram de maior suporte ventilatório (CPAP e ventilação mecânica), sugerindo o papel da prematuridade no Grupo (2) (HENTGES et al., 2010).

HENTGES et al., 2010, enfocam que o tratamento adequado de apneia no período neonatal é de suma importância, já que há maior incidência de hemorragia peri-intraventricular (HPIV), hidrocefalia, leucomalácia periventricular (LPV), necessidade de suporte ventilatório e alterações no desenvolvimento neurológico no primeiro ano de vida, quando os RNPT com apneia são comparados com os sem apneia.

Hiperbilirrubinemia é um dos mais frequentes diagnósticos em neonatos pré-termos. O diagnostico correto e precoce da hiperbilirrubinemia indireta pode reduzir as taxas de morbidade neonatal (LIMA et al., 2007). Diante da situação, LIMA et al., (2007), revisaram condutas clínicas por meio de um estudo de coorte retrospectivo para definir os fatores de risco associados a "hiperbilirrubinemia indireta neonatal" moderada ou grave. Em meio à amostra de 818 RN, os autores do trabalho analisaram um grupo de $94 \mathrm{RN}$ ictéricos que foram submetidos à fototerapia, estabelecendo a seguinte assertiva: a prematuridade $(52,1 \%$ de crianças com idade gestacional inferior a 37 semanas) influenciou a gravidade da icterícia, devido certamente ao metabolismo imaturo da bilirrubina. Eles confirmaram, ainda, que o baixo peso ao nascimento e a idade 
gestacional precoce $(\mathrm{p}=0.00)$ aumentaram a necessidade de fototerapia, não havendo, porém, correlação simultânea destes fatores com a gravidade da icterícia.

Espelhando-se em outros estudos nacionais e frente à necessidade epidemiológica da região foi desenvolvido no Vale do Paraíba Paulista, na região sudeste do Brasil, um estudo transversal com dados secundários obtidos na Secretaria Estadual da Saúde referentes aos nascimentos ocorridos em 2004. Anencefalia, encefalocele e espinha bífida (mielocele e mielomeningocele) foram considerados defeitos de fechamento do tubo neural por NASCIMENTO, (2008). Este analisou variáveis dos RN como escore de Apgar de 5 minutos, peso ao nascer $(\mathrm{g})$ e duração da gestação. As variáveis neonatais (baixo peso, prematuridade e baixa vitalidade) relacionaram-se com a anomalia. $\mathrm{O}$ risco de ocorrência do defeito de fechamento de tubo neural associado aos nascimentos prematuros foi em torno de seis vezes maior dentre os portadores de defeitos no tubo neu-ral. Ao comparar o grupo de RNPT com defeito de fechamento do tubo neural e os RNPT com ausência desse defeito o autor encontrou $\mathrm{p}<0,001$ e $\mathrm{OR}=5,80$ (2,70-12,20) (NASCIMENTO, 2008).

A Lesão da Substância Branca (LSB) é indicada como sendo a mais frequente lesão cerebral em RN prétermo e a termo. Com o objetivo de analisar a associação entre fatores natais com a gravidade da LSB cerebral neonatal, controlando o peso de nascimento, foi realizado na Bahia (Brasil), em um hospital terciário referência de atendimento às gestantes de alto risco, um estudo clínico-epidemiológico baseado em dados secundários. Os autores identificaram os neonatos pela ultra-sonografia craniana, sendo estes divididos em: aqueles com evolução da LSB para resolução da imagem ao ultra-som (menor gravidade) e, aqueles que evoluíram com formação de cistos e/ou ventriculomegalia e/ou hemorragia (maior gravidade). No período referente ao estudo 89 neonatos foram selecionados, 12 (13,5\%) foram a termo e, destes, oito tiveram peso de nascimento

\section{REFERÊNCIAS}

1. ARGOLLO N, LESSA I, RIBEIRO S, ABREU KC, PINTO JMS, FARIA RP, TELLES TG, SANTOS GB. Peso de nascimento como preditor para gravidade da lesão da substância branca cerebral neonatal. Arq Neuropsiquiatr. 2010; 64(2A):287-294.

2. BECK S, WOJDYLA LS, BETRAN AP, MERIALDI M, REQUEJO JH, RUBENS C, et al. The worldwide incidence of preterm birth: a systematic review of maternal mortality and morbidity. Bull World Health Organ. 2010; 88:31-8.

3. CAIXETA FF, CORREAA MSNP. Os defeitos do esmalte e a erupção dentária em crianças prematuras. Rev Assoc Med Bras. 2005; 51(4): 195-9. superior a $2.500 \mathrm{~g}$; entre os RNPT $11(12,4 \%)$ tiveram peso nesta faixa. A prematuridade foi a principal causa do baixo peso de nascimento em $77(86,52 \%)$, sendo os neonatos mais prematuros os de menores pesos. Através do teste do qui-quadrado a prematuridade indiciou diferença estatística significativa entre os grupos ( $\mathrm{p}=0,0004)$ (ARGOLLO et al., 2006).

A busca por artigos para realizar esta revisão demonstrou a escassez de estudos variados que objetivaram analisar a relação entre prematuridade e os riscos para o desenvolvimento de doenças em crianças ou a cronicidade em adultos (apontada no trabalho de JOHASSON et al, 2005). A maior parcela dos estudos encontrados referiam-se a ROP, provavelmente pela importância dos dados de incidência obtidos nos países subdesenvolvidos como exemplificado por LOMUTO et al., (2010). Muitas pesquisas associaram o baixo peso ao nascer como fator decorrente da imaturidade, demonstrando a forte ligação do tema.

\section{CONSIDERAÇÕES FINAIS}

Uma parte significativa dos artigos selecionados foi publicada em 2010, dado que evidencia ser - a prematuridade - um tema de interesse bastante recente. A relativa pequena quantidade de artigos, bem como a atualidade do tema, pode estar relacionada ao aumento da sobrevida de prematuros propiciado pelo avanço de tecnologias na área da saúde nos últimos tempos.

Evidencia-se a associação entre o tempo de gestação e o aumento do risco para o desenvolvimento de doenças cardiovasculares e outros agravos crônicos tanto em fases mais precoces da vida quanto em fases mais tardias (vida adulta). A prematuridade ocorre frequentemente em concomitância com o baixo peso, contribuindo para o desenvolvimento dos mais variados impactos, a exemplo da hiperbilirrubinemia.

4. CARLINO FC, LAMÔNICA DAC, ALVARENGA KF. Avaliação da função auditiva receptiva, expressiva e visual em crianças prematuras. Pró-Fono Revista de Atualização Científica. 2010; 22(1):19-24.

5. CHEUNG YF, WONG KY, LAM BC, TSOI NS. Relation of arterial stiffness with gestational age and birth weight. Arch Dis Child. 2004; 89:217-21.

6. COELLI AP, NASCIMENTO LR, MILL JG, MOLINA MCB. Prematuridade como fator de risco para pressão arterial elevada em crianças: uma revisão sistemática. Cad Saúde Pública. 2011; 27(2): 207-218. 
7. DALZIEL SR, PARAG V, RODGERS A, HARDING JE. Cardiovascular risk factors at age 30 following preterm birth. Int J Epidemiol. 2007; 36:907-15.

8. GROSSO LM, TRICHE E, BENOWITZ NL, BRACKEN MB. Prenatal caffeine assessment: fetal and maternal biomarkers or self-reported intake? Ann Epidemiol. 2008; 18:172-8.

9. HENTGES CR, GUEDES RR, SILVEIRARC, PROCIANOY RS. Serum levels of caffeine in umbilical cord and apnea of prematurity. J Pediatr. 2010; 86(2):137-142.

10. FELICE TD, RIBEIRO JAAB, ISHIZUKA ROR, GODOY GJ. Motor development in nine-month-old preterm infants. Med Reabil.2010; 29(1): 19-22.

11. JANVIER A, KHAIRY M, KOKKOTIS A, CORMIER C MESSMER D, BARRINGTON KJ. Apnea is associated with neurodevelopmental impairment in very low birth weight infants. J Perinatol.2004; 24:763-8.

12. JOHANSSON S, ILIADOU A, BERGVALL N, TUVEMO T, NORMAN M, CNATTINGIUS S. Risk of high blood pressure among young men increases with the degree of immaturity at birth. Journal of American Heart Association. 2005;112:3430-6.

13. LAWOR DA, HUBINETTE A, TYNELIUS O, LEON DA SMITH GD, RASMUSSEM F. Associations of gestational age and intrauterine growth with systolic blood pressure in a family-based study of 386485 men in 331089 families. Circulation. 2007:115:562-8.

14. LIMA GM, PORTO MASC, BARBOSAAP, CUNHAAJLA. Fatores de risco preditivos de hiperbilirrubinemia neonatal moderada a grave. Einstein. 2007; 5(4):352357.

15. LOMUTO CC, GALINA L, BRUSSAM, QUIROGAA, ALDA E, BENÍTEZAM, BOUZAS L, DINERSTEIN NA, FALBO J, MANZITTI J, MARINARO S, NIETO R, SEPÚLVEDA T, VISINTÍN P. Epidemiología de la retinopatía del prematuro em servicios públicos de la Argentina durante 2008. Arch Argent Pediatr. 2010;108(1):24-30.
16. LORENA SHT, BRITO JMS. Estudo retrospectivo de crianças pré-termo no Ambulatório de Especialidades Jardim Peri-Peri. Arq Bras Oftalmol. 2009; 72(3):360-4.

17. Brasil, Ministério da Saúde. Informações e análises. Uma análise dos nascimentos no Brasil e regiões. Disponível em: [http://portal.saude.gov.br/portal/saude/ Gestor/visualizar texto.cfm?idtxt=24455]. Acessado em: 16 de maio/2011.

18. NASCIMENTO LFC. Prevalência de defeitos de fechamento de tubo neural no Vale do Paraíba, SP. Rev Paul Pediatr. 2008; 26(4):372-7.

19. RABELLO MSC, BARROS SMO. Prematurity: clinical and epidemiological aspects in a Normal Delivery Center. Einstein. 2011; 9 (4 Pt 1):483-8.

20. SINCLAIR JC, BRACKEN MB, ATKINSON SA. Feeding the normal term infant: human milk and formula. In: Effective Care of the Newborn Infant (Sinclair JC, BrackenMB, eds). Oxford: Oxford University Press. 1992; 79-92.

21. TIAGO LF, CALDEIRA AP, VIEIRA MA. Risk factors for low birthweight in a public maternity of inner Minas Gerais State. Pediatria (São Paulo). 2008; 30(1):8-14.

\section{Correspondência}

Thassiany Sarmento Oliveira de Almeida

Endereço: Rua José Ulisses de Lucena, 215, Mirante.

Campina Grande - Paraíba - Brasil

CEP: 58407688

Email: thassysarmento@hotmail.com 